\title{
ERRATUM
}

\section{Erratum to: Urban Health Research in Africa: Themes and Priority Research Questions}

Tolu Oni, Warren Smit, Richard Matzopoulos, Jo Hunter-Adams, Michelle Pentecost, Hanna-Andrea Rother, Zulfah Albertyn, Farzaneh Behroozi, Olufunke Alaba, Mamadou Kaba, Claire van der Westhuizen, Maylene Shung-King, Naomi S. Levitt, Susan Parnell, and Estelle V. Lambert, RICHE members

\section{Erratum to: Journal of Urban Health} DOI: $10.1007 / \mathrm{s} 11524-016-0050-0$

Please note that the given name of coauthor Farzaneh Behroozi was misspelled in this article as originally published (it was spelled incorrectly as "Farzeneh").

Oni is with the Division of Public Health Medicine, School of Public Health and Family Medicine, University of Cape Town, Cape Town, South Africa; Smit and Parnell are with the African Centre for Cities, University of Cape Town, Cape Town, South Africa; Matzopoulos is with the Burden of Disease Research Unit, South African Medical Research Council, Cape Town, South Africa; Hunter-Adams and Alaba are with the Health Economics Unit, School of Public Health and Family Medicine, University of Cape Town, Cape Town, South Africa; Pentecost is with the Department of Anthropology, University of Cape Town, Cape Town, South Africa; Pentecost is with the Institute of Social and Cultural Anthropology, University of Oxford, Oxford, UK; Rother is with the Division of Environmental Health and Centre for Environmental and Occupational Health Research, School of Public Health and Family Medicine, University of Cape Town, Cape Town, South Africa; Albertyn is with the Children's Institute, Department of Paediatrics, University of Cape Town, Cape Town, South Africa; Behroozi is with the Primary Health Care Directorate, University of Cape Town, Cape Town, South Africa; Kaba is with the Division of Health Economics, School of Public Health and Family Medicine, University of Cape Town, Cape Town, South Africa; Kaba is with the Division of Medical Microbiology, Department of Clinical Laboratory Sciences, University of Cape Town, Cape Town, South Africa; van der Westhuizen is with the Alan J Flisher Centre for Public Mental Health, Department of Psychiatry and Mental Health, University of Cape Town, Cape Town, South Africa; Shung-King is with the Division of Health Policy and Systems, School of Public Health and Family Medicine, University of Cape Town, Cape Town, South Africa; Levitt is with the Chronic Disease Initiative for Africa and Division of Diabetic Medicine and Endocrinology, Department of Medicine, University of Cape Town, Cape Town, South Africa; Lambert is with the Division of Exercise Science and Sports Medicine, Department of Human Biology, University of Cape Town, Cape Town, South Africa.

Correspondence: Tolu Oni, Division of Public Health Medicine, School of Public Health and Family Medicine, University of Cape Town, Cape Town, South Africa. (E-mail: tolullah.oni@uct.ac.za) The online version of the original article can be found at doi:10.1007/s11524-016-0050-0. 